Memorias del VII Encuentro Nacional de Experiencias en la Enseñanza de la Biología y la Educación Ambiental y II Congreso Nacional de Investigación en la Enseñanza de la Biología

\title{
LA DEFENSA BIOLÓGICA PRESENTE EN LOS PROCESOS DE PARTICIPACIÓN EN EL AULA
}

\section{THE BIOLOGICAL DEFENSE PRESENT IN THE PROCESSES OF PARTICIPATION IN THE CLASSROOM}

\section{Zuleny Aguilera Martínez ${ }^{1}$}

\section{Eduardo Federico Segura Ávila²}

\section{Laura Marcela Trujillo Castro ${ }^{3}$}

\section{Resumen}

El eje curricular Dinámica y Mantenimiento de los Sistemas del Departamento de Biología de la Universidad Pedagógica Nacional plantea indispensable en la formación como Licenciados en Biología el diseño de una Unidad Didáctica (UD), en tanto permite identificar particularidades de contextos educativos colombianos para abordar conceptos específicos desde la enseñanza de la Biología. En ese sentido, el presente documento es resultado del diseño de una UD "Entre el Mundo de las Respuestas 7" con la cual se desarrolla la temática "defensa biológica", dirigida a estudiantes de grado séptimo de la jornada mañana de la Institución Educativa Distrital Guillermo Cano Isaza. Desde el ejercicio de contextualización y caracterización, ésta propuesta didáctica contempla que en los procesos de aprendizaje el estudiante debe tener una participación activa que le permita desempeñar un rol dinámico y significativo en el momento de construir y co-construir conocimientos relacionados con conceptos biológicos. Por consiguiente, bajo el enfoque sociocultural del modelo constructivista, se plantea y elaboran actividades y contenidos con los cuales se busca en primer lugar, fomentar la participación activa de los estudiantes y con ello el aprendizaje significativo a través de la enseñanza de los procesos de defensa del sistema vivo; y en segunda instancia, se pretende reflexionar sobre el ejercicio docente en el diseño, construcción y análisis de estrategias didácticas relacionadas con la enseñanza de la biología. Dicho ejercicio permitió enriquecer, retroalimentar y reflexionar el proceso de formación como Licenciados en Biología en el contexto Colombiano.

\footnotetext{
${ }^{1}$ Estudiante Licenciatura en Biología UPN dbi_zaguilera551@pedagogica.edu.co

2 Estudiante Licenciatura en Biología UPN dbi_esegura624@pedagogica.edu.co

${ }^{3}$ Estudiante Licenciatura en Biología UPN dbi_Itrujillo432@pedagogica.edu.co
} 
Bio-grafia Escritos sobre la Biologia y su Enseñanza.

Edición Extra-Ordinaria. ISSN 2027-1034 P.p 974 - 985

Memorias del VII Encuentro Nacional de Experiencias en la Enseñanza de la

Biología y la Educación Ambiental y II Congreso Nacional de Investigación en la Enseñanza de la Biología

\section{Palabras Clave}

Defensa Biológica, Unidad Didáctica, Participación Activa, Aprendizaje Significativo, Enseñanza de la Biología.

\section{Abstract}

The axis curricular Dynamics and Maintenance of the Systems of the Department of Biology of the Pedagogic National University raises indispensably in the formation as Licentiates in Biology the design of a Didactic Unit (DU), while it allows to identify particularities of educational Colombian contexts to approach specific concepts from the teach of the Biology. In this sense, the present document is a result of the design of one DU called "Between the World of the Defense" with which there develops the thematic " biological defense " directed students of the seventh degree of the day tomorrow of the Educational Institution Distrital Guillermo Cano Isaza. From the exercise of context and characterization, this one proposed didactics contemplates that in the learning processes the student must have an active participation that allows him to recover a dynamic and significant role in the moment to construct and co-construct knowledge related to biological concepts. Consequently, down the sociocultural approach of the model "constructivista", appears and we elaborate activities and contents with which it is looked first, to promote the active participation of the students and with it the significant learning across the teach of the processes of defense of the alive system; and in the second instance, one tries to think about the educational exercise in the design, construction and analysis of didactic strategies related to the teach of the biology. The above mentioned exercise allowed to enrich, and to think over the process of formation as Licentiates in Biology in the Colombian context.

Key Words: Biological defense, Didactic Unit, Active Participation, Significant Learning, Teach of the Biology.

\section{Introducción}

En la continua búsqueda de aquello que implica ser docente, el eje curricular Dinámica y Mantenimiento de los Sistemas del Departamento de Biología de la Universidad Pedagógica Nacional, plantea que el diseño de una Unidad Didáctica (UD) fundamentada en particularidades de los contextos educativos, y por ende en los procesos de enseñanza-aprendizaje de la biología actuales permite al futuro docente elaborar estrategias pertinentes, fundamentadas y contextualizadas para lograr la reflexión de su quehacer, y que el aprendizaje sea significativo para los estudiantes. Para ello, es necesario captar su atención, sin embargo, puede ser difícil en muchos casos debido a los múltiples elementos distractores a los que el 
Bio-grafia Escritos sobre la Biologia y su Enseñanza.

Edición Extra-Ordinaria. ISSN 2027-1034 P.p 974 - 985

Memorias del VII Encuentro Nacional de Experiencias en la Enseñanza de la

Biología y la Educación Ambiental y II Congreso Nacional de Investigación en la Enseñanza de La Biología

estudiante está expuesto en el aula. Lo cual se complejiza, puesto que según Cruz (2003) las diferentes formas en que un maestro pudiera presentar su clase pueden resultar poco atractivas y no motivar el aprendizaje por parte del alumno. Es aquí donde el docente, según Camilloni (2007), debe cuestionar las modalidades de enseñanza, lo cual implica repensar el modelo tradicional de transmisión donde no se tienen en cuenta las ideas previas de los estudiantes, y no se pretenden alcanzar aprendizajes significativos por parte de estos.

Es así, que el diseño y realización de la UD concerniente al patrón defensa surge de cuestionar y problematizar una práctica pedagógica en la cual los estudiantes no presentan un papel activo y participativo en sus procesos de aprendizaje, perjudicando la construcción de conocimientos, pues estos actúan como espectadores de una presentación hecha por el maestro sin opinar, juzgar y decidir de forma colectiva sobre su propia formación. En consecuencia, surge la siguiente situación problema, que permite el diseño y elaboración de la UD ¿Cómo generar acciones participativas en los estudiantes de grado séptimo a partir del aprendizaje de los procesos de defensa del sistema vivo?

Para tal fin, se establecen objetivos específicos buscando: desarrollar habilidades comunicativas entre los estudiantes como estrategia participativa; identificar los procesos de defensa que desarrolla el sistema vivo; y fomentar la participación activa de los estudiantes a través de las actividades planteadas en la Unidad Didáctica referentes al trabajo en equipo.

En ese sentido, con el diseño y realización de la UD "Entre el mundo de las respuestas 7", el estudiante es motivado a participar en la construcción de su propio conocimiento debido a que aquel tendrá la oportunidad de contemplar y estudiar procesos de defensa del sistema vivo a través de actividades centradas en el trabajo en equipo, ideas previas, intercambio de conocimientos, relaciones e interacciones con los otros sujetos en pro de la construcción de conocimientos significativos. Pues, como menciona Hernández (2008) los procesos de enseñanza-aprendizaje son una construcción conjunta entre sujetos, por tanto, las actividades de la UD se caracterizan por contemplar a los otros (adultos o pares) con quienes se interrelaciona, intercambia e interviene activamente en los procesos de construcción del conocimiento convirtiéndose en auténticos coprotagonistas o co-constructores.

La UD permite al maestro implementar actividades que apoyen o medien de forma contextualizada y coherente su acción, deja de ser el eje del aprendizaje que se realiza en clase, abre nuevas vías y posibilidades de enseñanza-aprendizaje, debido a que, transfiere una parte de la responsabilidad por aprender a los alumnos, conllevando a estos a tener un papel más participativo, autónomo y relevante en su formación. De igual forma, para el docente en formación permitió 
Bio-grafia Escritos sobre la Biologia y su Enseñanza.

Edición Extra-Ordinaria. ISSN 2027-1034 P.p 974 - 985

Memorias del VII Encuentro Nacional de Experiencias en la Enseñanza de la

Biología y la Educación Ambiental y II Congreso Nacional de Investigación en la Enseñanza de la Biología

trabajar en equipo, fomentar la cooperación, creatividad y confianza con sus pares lo cual contribuyo a consolidar y cimentar la profesión docente que involucra aspectos conceptuales, metodológicos y actitudinales. Finalmente, la UD "Entre el mundo de las respuestas 7" brinda elementos para el ejercicio docente en el aula abre las puertas para la creatividad y la innovación en las vías de ser maestro ante un realidad cambiante e inacabada como lo es la escolar, en el contexto Colombiano.

\section{Metodología}

El diseño de la UD "Entre el mundo de las respuestas 7", se desarrolló en cuatro fases, descritas a continuación.

Fase 1 contextualización y caracterización: Se realiza un acercamiento a aspectos sociales, educativos y culturales a nivel local, institucional y del curso a través de consultas bibliográficas; visitas al barrio y a la institución; entrevistas con estudiantes y docentes; observación no participante en el aula; y la implementación de cuestionarios para reconocer la población y sus ideas sobre el concepto de defensa biológica.

Fase 2 construcción: Atañe al planteamiento de la pregunta problema teniendo en cuenta lo experimentado en la fase 1; construcción de objetivos formativos, disciplinares y actitudinales, justificación desde el eje curricular, búsqueda de antecedentes contemplando investigaciones realizadas sobre la temática a nivel internacional, nacional y de la Universidad Pedagógica Nacional; y la construcción del marco teórico centrado en las ideas centrales de la propuesta didáctica, estableciendo conceptos estructurantes.

Fase 3 diseño: Se lleva a cabo el diseño de la UD, teniendo en cuenta la construcción de contenidos y actividades. Así, los contenidos se disponen de tal manera que responden al ¿qué? enseñar; por consiguiente, los contenidos conceptuales se refieren al "saber" o al "saber decir", los procedimentales implican el aprendizaje del "saber hacer", y los actitudinales se refieren al "sentir". Por su componente cognitivo, afectivo y evaluativo, se refieren a las normas, actitudes y valores que pueden aprender los estudiantes (IBER, 1995).

Fase 4 resultados: Análisis y reflexión sobre el ejercicio del diseño de la UD, planteada desde las perspectivas, pedagógica, didáctica y disciplinar. 
Bio-grafia Escritos sobre la Biologia y su Enseñanza.

Edición Extra-Ordinaria. ISSN 2027-1034 P.p 974 - 985

Memorias del VII Encuentro Nacional de Experiencias en la Enseñanza de la

Biología y la Educación Ambiental y 11 Congreso Nacional de Investigación en la Enseñanza de la Biología

\section{Resultados Y Discusión}

Los resultados del presente proyecto, se presentan en dos etapas.

\section{Etapa 1. Construcción del Documento Formativo}

La construcción del documento formativo LA DEFENSA BIOLÓGICA PRESENTE EN LOS PROCESOS DE PARTICIPACIÓN EN EL AULA retoma la caracterización de la población a la cual va dirigida la UD, el planteamiento de la problemática evidenciada, los objetivos de la UD y objetivos formativos, la justificación del proyecto desde aspectos sociales, políticos, económicos, culturales, disciplinares y educativos, la construcción de un marco teórico referente al patrón defensa, a los aspectos pedagógicos, didácticos, y políticos (lineamientos propuestos por el Ministerio de Educación Nacional).

Por otro lado, se realizó una consulta de antecedentes relacionados con el diseño de UD y libros de Ciencias Naturales (CN) utilizados por los maestros en las escuelas, los cuales aportaron al presente proyecto, en la medida en que exponen que la enseñanza de las $\mathrm{CN}$, en este caso de Biología, debe abordarse de forma que los estudiantes interpreten y comprendan su entorno haciendo uso de los aprendizajes construidos en la interacción con los sujetos inmersos en el aula, Igualmente, se pretende que los estudiantes no solo desarrollen un aprendizaje de conceptos, sino que también fortalezcan habilidades procedimentales y actitudinales para su formación personal.

Igualmente, se retoman algunos planteamientos de la Teoría General de los Sistemas (TGS), los cuales exponen que un sistema presenta una características particulares como, la estructuración de redes de relaciones entre las partes del sistema, la aparición de distintos niveles sistémicos (o subsistemas), y la emergencia de procesos mediante los cuales el sistema se mantiene dentro de determinados rangos que permiten su subsistencia (Capra, 1998).

A partir de los marcos conceptuales de la TGS, el eje curricular Dinámica y Mantenimiento de los Sistemas se propone la construcción de unas redes de relaciones para abordar el diseño de la unidad didáctica, dichas redes deben dar cuenta de tres aspectos: el primero es la red disciplinar en la cual se retoma el patrón de organización defensa, en segundo lugar se encuentra la red pedagógico-didáctica; y finalmente se desarrolla una red de integración disciplinarpedagógico-didáctica o también conocida como red de transposición didáctica.

Cabe resaltar que para el diseño de la red disciplinar-pedagógico-didáctica se tiene en cuenta la definición de transposición didáctica propuesta por Chevallard, Y (1998), entendida como la transformación de un saber sabio hacia un saber 
Bio-grafia Escritos sobre la Biología y su Enseñanza.

Edición Extra-Ordinaria. ISSN 2027-1034 P.p 974 - 985

Memorias del VII Encuentro Nacional de Experiencias en la Enseñanza de la

Biologia y la Educación Ambiental y II Congreso Nacional de Investigación en la Enseñanza de la Biología

enseñado, el cual este contextualizado con las dinámicas de la realidad, dicha transposición didáctica no es la simplificación del conocimiento sino la revisión de aquel conocimiento que se lleva al aula. En ese sentido, para el diseño de la red de relaciones es necesario reflexionar acerca de qué, cómo, para qué y por qué se va a enseñar una temática determinada.

\section{Etapa 2. Construcción de la Unidad Didáctica}

La Construcción de la Unidad Didáctica ENTRE EL MUNDO DE LAS RESPUESTAS 7, es el resultado de la elaboración del documento formativo en el cual se realizaron diferentes revisiones bibliográficas y la contextualización, que posibilitaron el diseño de una UD, la cual aborda la temática del patrón Defensa.

La UD, consta de contenidos los cuales responden al qué enseñar, desde lo conceptual, lo procedimental y lo actitudinal, que tienen por fin, desarrollar las capacidades las cuales se organizan de acuerdo a la dificultad de los aprendizajes, la edad, los intereses y las ideas previas de los estudiantes.

Por consiguiente, para el diseño de la Unidad didáctica se proponen los siguientes contenidos temáticos:

\section{CAPÍTULO 1: Diversidad y clasificación de seres vivos}

TEMA 1: Características de los sistemas vivos.

TEMA 2: ¿Qué es diversidad y clasificación?

TEMA3: La diversidad de organismos y su clasificación.

En éste capítulo, se exponen las características de aquello que se considera como organismo vivo, y cómo éstos se clasifican. El capítulo busca, que los sujetos consoliden actitudes de respeto frente a otros organismos.

\section{CAPÍTULO 2: Estímulos y respuestas}

TEMA 1: ¿Qué son estímulos y respuestas?

TEMA 2: ¿Cómo responden los grupos de organismos a los estímulos?

Éste capítulo, presenta las características de los conceptos estímulo y respuesta, y la manera en que los organismos responden a los diferentes estímulos del entorno

\section{CAPÍTULO 3: Defensa}

TEMA 1: ¿Qué es defensa?

TEMA 2: ¿Cómo se defienden los grupos de organismos? 
Bio-grafia Escritos sobre la Biologia y su Enseñanza.

Edición Extra-Ordinaria. ISSN 2027-1034 P.p 974 - 985

Memorias del VII Encuentro Nacional de Experiencias en la Enseñanza de la

Biología y la Educación Ambiental y II Congreso Nacional de Investigación en la Enseñanza de la Biología

El capítulo 3, aborda los procesos en que los organismos se defienden frente a los distintos estímulos del medio.

Cabe mencionar que a partir del desarrollo de los tres capítulos, se realiza una revisión de cómo ocurren dichos procesos en microorganismos, plantas y animales, para que el estudiante compare e identifique las diferencias y similitudes entre los procesos que se llevan a cabo en los organismos que tienen como finalidad defenderse ante un estímulo.

Finalmente, se formula la Temática $\boldsymbol{Y}$ ¿en la actualidad?, la cual está enfocada a la relación entre el patrón de defensa, el contexto y la cultura, para evidenciar la interacción de los aprendizajes con éstos, para que así el estudiante relacione el concepto de defesa con situaciones problema que identifique en su entorno.

De acuerdo a lo anterior, se establece una secuenciación, entendida como la temporalizarían de los contenidos y criterios de evaluación a lo largo de los ciclos de la etapa y responde al "¿cuándo?" enseñarlos. Es decir, para el diseño de la UD, se establece una organización y secuencia de contenidos teniendo en cuenta los conceptos estructurantes y los conceptos específicos de cada capítulo.

En cuanto a las actividades, las cuales según, IBER (1995) son un conjunto de acciones con coherencia interna que se organiza para que los estudiantes organicen sus habilidades, mediante los contenidos conceptuales, procedimentales y actitudinales; en la UD se plantean actividades que posibiliten el proceso de enseñanza-aprendizaje a partir de: actividades iniciales, para explicitar las ideas preconcebidas, actividades de reestructuración las cuales permiten un cambio conceptual a partir de la reconstrucción de sus conocimientos, actividades de aplicación, cuyo objetivo es reforzar el aprendizaje y actividades de revisión, que posibilitan la revisión de las construcciones que se realizan entre los sujetos inmersos en el aula durante el proceso (IBER, 1995). Dichas actividades se desarrollan a lo largo de la UD, las cuales retomaran los aspectos conceptuales, procedimentales y actitudinales.

Finalmente, se establece la evaluación como una estrategia pedagógica debido a que regula los procesos de enseñanza-aprendizaje durante la actividad educativa, de esta manera se retoma la evaluación inclusiva que toma elementos del constructivismo, la cual se caracteriza por: Tareas realistas y contextualizadas, Reflexión y valoración del alumno. Igualmente, se tiene en cuenta la evaluación constructivista, que según IBER (1995) se establece cundo el estudiante participa de las decisiones en el proceso de enseñanza-aprendizaje, comprometiéndose con su aprendizaje, autoevaluándose, evaluando a sus compañeros y al proceso, permitiendo la retroalimentación del mismo. En síntesis, se asume que la evaluación es un proceso que permite la reflexión del proceso educativo en la 
Bio-grafia Escritos sobre la Biologia y su Enseñanza.

Edición Extra-Ordinaria. ISSN 2027-1034 P.p $974-985$

Memorias del VII Encuentro Nacional de Experiencias en la Enseñanza de la

Biología y la Educación Ambiental y II Congreso Nacional de Investigación en la Enseñanza de la Biología

cual, no se esperan resultados perfectos, por el contrario se asumen los resultados en los que se evidencien aspectos por mejorar y en los que se identifique hasta qué punto fue el cumplimiento de los objetivos propuestos para la UD y para la formación como futuros Licenciados en Biología, permitiendo una retroalimentación en la práctica docente.

\section{Conclusiones}

El diseño de la Unidad Didáctica ENTRE EL MUNDO DE LAS RESPUESTAS 7, contribuye a la formación como Licenciados en Biología, debido a que se otorga importancia al reconocimiento del contexto en el que el maestro colombiano está inmerso, y en el cual convive con diferentes sujetos mediante las interacciones que se gestan día a día. Es así, como el maestro logra ubicarse en el entorno como aquel sujeto generador de relaciones, las cuales pueden aportar a la transformación de las problemáticas actuales de Colombia

Por consiguiente, las inter-relaciones que se entablan en la escuela en el proceso de enseñanza-aprendizaje, permiten reconocer que el maestro no tiene lugar únicamente en el aula, sino en las acciones cotidianas en los espacios del compartir y de escucha de los aportes del otro. Por ende, la UD posibilitó indagar acerca de cómo lograr la convergencia de dichas experiencias en el espacio académico, es así, como se logra manifestar que gracias a la didáctica, reconocida como una disciplina autónoma, pero que bebe de otras como la pedagogía y la psicología, permite la socialización de los conocimientos cotidianos y de los conocimientos científicos, para posibilitar la emergencia del conocimiento escolar a partir de las construcciones y transformaciones conceptuales, al tener en cuenta las ideas expresadas por los compañeros y el profesor. Así mismo, el diseño de la UD, aporta en el reconocimiento de los conocimientos procedimentales y actitudinales de los estudiantes, puesto que estos dos factores influyen en la comprensión de determinadas temáticas.

Igualmente, se concluye que es fundamental enmarcarse dentro de un enfoque pedagógico, debido a que éste orienta la coherencia de la práctica docente, es decir, permite articular el discurso con la acción del quehacer del maestro. Por ende, para el proyecto se escoge el Constructivismo Social fundamentalmente porque plantea que la reestructuración del conocimiento no es netamente individual, sino que es el producto de las relaciones e interacciones con otros sujetos, permitiendo que el conocimiento trascienda del individuo a la sociedad. Por lo tanto, es indispensable tenerlo en cuenta para el diseño de las actividades presentes en Unidad didáctica, en tanto que posibilita las intenciones educativas del profesor. 
Bio-grafia Escritos sobre la Biologia y su Enseñanza.

Edición Extra-Ordinaria. ISSN 2027-1034 P.p 974 - 985

Memorias del VII Encuentro Nacional de Experiencias en la Enseñanza de la

Biología y la Educación Ambiental y II Congreso Nacional de Investigación en la Enseñanza de La Biología

Por otro lado, en cuanto al aspecto disciplinar se logra comprender que los seres vivos llevan a cabo diferentes procesos para mantener el sistema dentro de determinados rangos de tolerancia, evitando que el organismo colapse y muera. Es así, como uno de dichos procesos es la Defensa, la cual, a partir de diversas indagaciones, revisiones, consultas y construcciones, es posible determinarla como: Los procesos que llevan a cabo los organismos vivos ante diferentes estímulos producidos por el medio, los cuales ponen en peligro el equilibrio dinámico de los sistemas. Dichos procesos se entienden como una serie de sucesos, que inician con la percepción del estímulo por medio de la membrana celular, el cual contiene un umbral, entendido como el mínimo grado de intensidad para desestabilizar el organismo y dar paso a que éste responda ante las fluctuaciones del medio. Estos procesos se desarrollan al interior de todos los seres vivos. Así mismo, se reconoce que a pesar de que la defensa es un patrón, cada grupo de organismos, animales, plantas y microorganismos, responden a partir de diferentes procesos a los estímulos de su entorno.

Finalmente, a partir del proceso del diseño de la Unidad Didáctica se concluye que es importante la perspectiva sistémica, en la que cada relación que se establece en los organismos es fundamental para mantener la emergencia de la vida. Por ende, el equipo de trabajo se aparta de la concepción antropocéntrica, la cual sostiene que el hombre es el centro del universo y por ende, todo debe confluir en él. En consecuencia, la postura de los autores es reconocernos como seres vivos sistémicos donde nuestras prácticas educativas y personales se enfocan en el reconocimiento, respeto y diálogo con la diversidad.

\section{Bibliografía}

Camilloni, A. (2007). El saber didáctico. Buenos Aires: Editorial Pardos.

Capra, F. (1998). La trama de la vida .Barcelona: Editorial Anagrama S.A

Chevallard, Y. (1998). La transposición didáctica. Del saber sabido al saber enseñado. Tercera Edición. Argentina: Aque.

Cruz, A. (2003). Propuesta Didáctica: Usos de Estrategias de Aprendizaje Cooperativo Para la Enseñanza de la Biología en Estudiantes de Educación Media Superior de la Universidad Autónoma de Nuevo León. (U.A.N.L.). Tesis. México. Recuperado el 15 de Abril del 2013 de http://eprints.uanl.mx/2395/1/1020149429.PDF

Hernández, G. (2008). Los Constructivismos y sus Implicaciones para la Educación. Vol. XXX, Núm. 122. Universidad Nacional Autónoma de México. 
Bio-grafía Escritos sobre La Biología y su Enseñanza.

Edición Extra-Ordinaria. ISSN 2027-1034 P.p 974 - 985

Memorias del VII Encuentro Nacional de Experiencias en la Enseñanza de la Biología y la Educación Ambiental y 11 Congreso Nacional de Investigación en la Enseñanza de la Biología

México, D.F., México. Redalyc. Recuperado el 15 de Abril del 2013 de http://www.redalyc.org/articulo.oa?id=13211181003

IBER, (1995). Diseño y Unidades Didácticas. Barcelona: Graò Educación.

\section{BIBLIOGRAFÍA RECOMENDADA}

- Adúriz, A \& Izquierdo, M (2002). Acerca de la didáctica de las ciencias como disciplina autónoma.

- Agrios, G. (1985). Fitopatología. México: Editorial Limusa.

- Alcaldía mayor de Bogotá. Secretaria de educación Ciudad Bolívar (s.f.). Recuperado el 18 de agosto del 2011 de http://matriculabd1.redp.edu.co/sistemat02/sed/directorio/ciudadbolivar.pdf

- Amador, P., Ávila, A., Gaytán, C., Domínguez, M. \& González, A. (2006). Maestría en Educación Antología Diseño Curricular. Secretaría de Educación y Cultura. Centro de Investigación y Docencia. Chihuahua, México. Recuperado el 23 de Mayo del 2012 de http://iinnuar.files.wordpress.com/2010/02/maestriaeducacion-disec3b1o-curricular-mex.pdf

- Apuntes de psicología. (2007). Apuntes de psicología. Recuperado el 3 de Abril de 2012, de http://www.apuntesdepsicologia.com/psicoanalisis/mecanismos-dedefensa.php

- Aravena, M (2006). Investigación educativa I. Recuperado el 24 de Mayo de 2012 de http://jirvargas.files.wordpress.com/2009/11/investigacion-educativa.pdf

- Bejarano, C., Castelblanco, Y., Eslava, E. \& Nieto J. (2006). Portal de la Ciencia. Ciencias Naturales y Educación Ambiental en el Noveno Grado. Colombia: Grupo Editorial Norma.

- Bonals, J. (2000). El trabajo en pequeños grupos en el aula. España: Editorial Graó.

- Campbell N, Reece J. (2007). Biología. (7 ed.). Madrid, España. Medica panamericana S.A.

- CEDID Guillermo Cano Isaza. (s.f.). Recuperado el 19 de agosto del 2011 de http://cedidferneisaza.blogspot.com/ 
Bio-grafia Escritos sobre la Biologia y su Enseñanza.

Edición Extra-Ordinaria. ISSN 2027-1034 P.p 974 - 985

Memorias del VII Encuentro Nacional de Experiencias en la Enseñanza de la Biología y la Educación Ambiental y 11 Congreso Nacional de Investigación en la Enseñanza de la Biología

- Claros, J. (s.f.). República de Colombia, contraloría de Santafé de Bogotá DC, Ciudad Bolívar, agenda de participación ciudadana. Grupo de investigación de la división de participación ciudadana.

- De Longhi, A. (s.f. ). ¿Cuáles son los principales cambios en la didáctica de la biología en los últimos años?. España. Recuperado el 12 de Mayo de 2012, de http://www.adbia.com.ar/cedivi recuros/cedivi/Memorias/Paneles\%20PDF/Panel\% 201\%20Panelista\%201.pdf

- De Probueno, A. (1999). Planificación de unidades didácticas por los profesores: análisis de tipos de actividades de enseñanza.

- Díaz, F (2002). Didáctica y Currículo: Un Enfoque Constructivista. España: Colección humanidades. Disponible http://books.google.com.co/books?id=Xrupzjit1 hkC\&printsec=frontcover\&dq=curric ulo\&hl=es\&sa $=X \&$ ei $=80$ e8T7jvNILUgQe48mqDw\&ved=0CDsQ6AEwAQ\#v=onepage\& $\mathrm{q}=$ curriculo\& $\mathrm{f}=$ false

- Embid, A. (s.f.). Resistencia de las bacterias a los antibióticos. Recuperado el 20 de Mayo de 2012 de http://www.amcmh.org/PagAMC/medicina/articulospdf/53ResistenciaBacterias.pdf

- Fernández, G \& Elortegui E. (1996). Investigación y Experiencias Didácticas, que piensan los profesores acerca de cómo se debe enseñar. España.

- Flórez, R. (1994). Hacia una Pedagogía del Conocimiento. Colombia: Editorial McGRAW-HILL.

- García, C. (1999). Como conocen los profesores la materia que enseñan. Algunas contribuciones de la investigación sobre conocimiento didáctico del contenido. España: Universidad de Sevilla

- Gómez, G \& Coll, C. (1993). ¿De qué hablamos cuando hablamos de constructivismo? Cuadernos de pedagogía.

- Gonzales, Á. (s.f). Recuperado el 12 de Abril del 2012 de http://www.inmunologiaenlinea.es/index.php?option=com_content\&view=article\&id $=102$ :filogenia\&catid=48:filogenia\&ltemid $=126$

- González, A. (s.f.). Filogenia sistema inmune. Recuperado el 20 de Mayo de 2012 de http://www.uco.es/grupos/inmunologiamolecular/inmunologia/tema16/etexto16.htm 
Bio-grafía Escritos sobre La Biología y su Enseñanza.

Edición Extra-Ordinaria. ISSN 2027-1034 P.p 974 - 985

Memorias del VII Encuentro Nacional de Experiencias en la Enseñanza de la Biología y la Educación Ambiental y 11 Congreso Nacional de Investigación en la Enseñanza de la Biología

- Habermas, J. (1987). Teoría de la acción comunicativa. Madrid: Taurus.

- Maier, R. (2001). Comportamiento animal. Un enfoque evolutivo y ecológico. España: Mc Graw Hill.

- Male, D. Brostoff, J. B.Rhot. D \& Roitt, I. (2007). Inmunología. España: Editorial Elsebier Mosby. Recuperado el 20 de Mayo de 2012 de http://books.google.com.co/books?id=JOhOzzZxDt4C\&printsec=frontcover\&dq=in munologia\&hl=es\&sa $=X \&$ ei $=\mathrm{I}-$

28T6y3K8P0ggfipb38Dg\&ved=0CDoQ6AEwAQ\#v=onepage\&q=inmunologia\&f=fal se

- Martínez, R. (s.f.). Historia de la inmunología. Datos cronológicos relevantes. Recuperado el 15 de Mayo de 2012 de http://www.tuobra.unam.mx/publicadas/021031121605.html

- Ministerio de Educación Nacional. MEN. (1998). Serie lineamientos curriculares.

- Ministerio de Educación Nacional. MEN. (s.f.). Ley 115 de 1994. Recuperado el 22 de Mayo de 2012 de http://menweb.mineducacion.gov.co/normas/concordadas/Decreto115.htm

- ORT Campus Virtual. (2012). CREA Centro de Recursos para la Enseñanza y el Aprendizaje. Argentina. Recuperado el del 2012 de http://campus.ort.edu.ar/crea/unidades didacticas

- Pacheco, J. (s.f.) Los mecanismos de la resistencia microbiana. Recuperado el 20 de Mayo de 2012 de http://www.ucsm.edu.pe/ciemucsm/larev/mecre.htm

- Parga, D., Ibarra, J. \& Mora W. (2000). Nuevo Investiguemos, Ciencias Naturales y de la Salud 6. Colombia: Editorial Voluntad

- Peña, J \& Cabello, A. Introducción a la inmunología. (s.f). Recuperado el 24 de Mayo del 2012 de http://www.inmunologiaenlinea.es/index.php?option=com content\&view=article\&id $=52$ :introduccion\&catid=37:introduccion-a-la-inmunologia\&ltemid=126

- Porlán, R. (1998). Pasado, presente y futuro de la didáctica de las ciencias.

- Quintanilla, M., Daza, S. \& Merino, C. (2010). Unidades Didácticas en Biología y Educación Ambiental. Su contribución a la promoción de competencias de 
Bio-grafia Escritos sobre La Biología y su Enseñanza.

Edición Extra-Ordinaria. ISSN 2027-1034 P.p 974 - 985

Memorias del VII Encuentro Nacional de Experiencias en la Enseñanza de la Biología y la Educación Ambiental y 11 Congreso Nacional de Investigación en la Enseñanza de la Biología

pensamiento científico. Volumen 4. Colombia: Grecia. Disponible en http://www7.uc.cl/sw educ/educacion/grecia/plano/html/pdfs/destacados/LibroDBio Grecia11julio.pdf

- Sánchez, G \& Valcárcel, M. (1993). Diseño de Unidades didácticas en el área de Ciencias Experimentales. España.

- Sanmarti, N. (2005) El diseño de unidades didácticas.

- Santillana (2010). Hipertexto Ciencias 7. Colombia: Editorial Grupo Santillana.

- Torres, G. (2008).Cuerpos extraños. Recuperado el 20 de Mayo de 2012 de http://www.santillana.com.co/websantillana/libros/hipertexto-ciencias-7/

- Uribe, M. (2009). Conociendo la localidad de ciudad bolívar. Diagnóstico de los aspectos físicos, demográficos y socio económicos. Secretaria distrital de planeación Bogotá $D$ C. Recuperado el 17 de agosto del 2011 de http://www.sdp.gov.co/resources/19ciudad bolivar.pdf

- Valbuena, E., Gutiérrez, A., Correa, M. \& Amórtegui, E. (2009). Formación inicial de profesores de biología en el marco del conocimiento Profesional del profesor II -Hallazgos-. Recuperado el 12 de Mayo de 2012 de http://www.pedagogica.edu.co/revistas/ojs/index.php/TED/article/viewArticle/231 